\title{
Liposomal Drug Delivery for Solubility and Bioavailability Enhancement of Efavirenz
}

\author{
MONICA R. P. RAO* AND LAXMI S. BABREKAR \\ Department of Pharmaceutics, AISSMS College of Pharmacy, near RTO, Kennedy Road, Pune-411 001, India
}

Rao and Babrekar: Liposomal Drug Delivery

\begin{abstract}
To overcome the limited solubility and low bioavailability of efavirenz a liposomal drug delivery system was formulated using thin film hydration technique. Optimal ratios of total lipid blend:drug, soya lecithin:cholesterol and polyethylene glycol 400 concentration were determined using Box-Behnken design with vesicle size and entrapment efficiency as responses. The optimized liposomal dispersions were characterized by vesicle size, entrapment efficiency, transmission electron microscopy, in vitro drug release and in vivo pharmacokinetics. The vesicle size was found to be in range of 694.5-1200.0 nm and entrapment efficiency was above $80 \%$. Statistical studies revealed that vesicle size and entrapment efficiency increased with increase in total lipid blend:drug and polyethylene glycol 400 concentration. Transmission electron microscopy showed that unilamellar and multi-lamellar vesicles were formed. Optimized liposomal dispersion was solidified using nanosponges. Solid liposomes were characterized by micromeritics, differential scanning calorimetry, Fourier-transform infrared spectroscopy and bioavailability. As compared to plain drug a 10-fold increase in percent release was observed in $6 \mathrm{~h}$ in liposomal preparation. In vivo pharmacokinetic studies revealed that bioavailability increases 2 folds as compared to plain drug. Lipid-based drug delivery like liposomes are taken up through lymphatic pathway. Since the human immunodeficiency virus settles in lymphoid organs, lymphatic drug delivery can be advantageous in the treatment of acquired immune deficiency syndrome. Thus, the pharmacokinetic studies demonstrated that efavirenz-loaded liposomes could significantly upgrade the solubility and oral bioavailability of efavirenz and improve the therapeutic efficacy.
\end{abstract}

Key words: Bioavailability, solubility, liposome, nanosponges, efavirenz

Solubility, dissolution and gastrointestinal permeability are fundamental parameters that control rate and extent of drug absorption and its bioavailability. Therefore, development of novel drug delivery systems are essential as a large number of new chemical drug entities have poor solubility or permeability. Solubility and bioavailability significantly influence attainment of desired concentration of drug in systemic circulation for generating a pharmacological action ${ }^{[1]}$.

The human immunodeficiency virus (HIV) causes one of the deadliest diseases of modern times, acquired immune deficiency syndrome (AIDS) affecting around 40 million people globally. HIV is a retrovirus of the lentivirus family, which fundamentally destroys $\mathrm{CD}_{4}^{+} \mathrm{T}$ cells a key segment of the immune system. Since 1996, the highly active antiretroviral treatment (HAART) has saved about 2.9 million lives ${ }^{[2]}$. Three main classes of antiretroviral (ART) drugs i.e. non-nucleoside reverse transcriptase inhibitors (NNRTIs), protease inhibitors,

*Address for correspondence

E-mail: monicarp_6@hotmail.com

November-December 2018
HIV integrase inhibitors are proposed in HAART regimens These include nevirapine, efavirenz (EFA), etravirine, rilpivirine, saquinavir, indinavir, ritonavir, raltegravir, elvitegravir, and dolutegravir.

Various drug delivery systems are used to deliver the HIV drugs in a controlled and targeted manner thereby increasing the drug bioavailability and residence time at target sites with a significant improvement in quality of life of HIV patients. These drugs are cost effective with less dose dumping problems. However, the ARTs have low oral bioavailability and cytochrome P450 (CYP)mediated metabolism ${ }^{[3]}$. The methodologies, which are used to overcome these drawbacks include chemical

This is an open access article distributed under the terms of the Creative Commons Attribution-NonCommercial-ShareAlike 3.0 License, which allows others to remix, tweak, and build upon the work non-commercially, as long as the author is credited and the new creations are licensed under the identical terms

Accepted 14 October 2018

Revised 06 April 2018

Received 03 July 2017

Indian J Pharm Sci 2018;80(6):1115-1124 
modification of pre-existing entities, reduction of vesicle size using high pressure homogenization, colloidal mill, development of novel delivery systems such as self-nano emulsifying drug delivery systems, solid-lipid nanoparticles, nanosponges and liposomes to improve the efficacy of ART drugs ${ }^{[4]}$.

Liposomes are microscopic vesicles comprising of one or more concentric spheres of lipid bilayer isolated by water or aqueous buffer compartment. Because of their biphasic features, diversity in design and composition, they are used as carriers for improving drug solubility, bioavailability, stability and targeting ${ }^{[5]}$. Liposomes have been used as a carrier for amphotericin B, silymarin, fenofibrate, dapsone, budesonide and artemisinin ${ }^{[6-10]}$. Liposomes can carry the drug in three potential compartments; water-soluble drugs in the central core, lipid-soluble drugs in the membrane, peptide and small proteins at the lipid-aqueous interface ${ }^{[11]}$. EFA is one of the widely used first-line NNRTI utilized in the pharmacotherapy of both geriatric and pediatric patients. It has high lipophilicity (log P:5.4) ${ }^{[12]}$ and poor aqueous solubility $(0.0085 \mathrm{mg} / \mathrm{ml})$, which makes EFA to be categorized as a BCS class II, having an oral bioavailability of $40-45 \%$ and relatively high intra (55-58 \%) and inter (19-24\%) subject variability. EFA is available in flexible doses as a tablet $(600 \mathrm{mg})$ and capsules (50 and $200 \mathrm{mg})^{[13]}$.

Previously, self-micro emulsifying drug delivery system has been developed using Acconon MC-8 EP, Cremophor EL and polyethylene glycol 400 (PEG 400) to enhance solubility and bioavailability of EFA ${ }^{[14]}$. Solid-lipid nanoparticles were formulated using glycerylmonostearate and Tween 80 as surfactant ${ }^{[15]}$. Nanosponges were developed using beta-cyclodextrin $(\beta-C D)$ and diphenyl carbonate to improve solubility and bioavailability of EFA ${ }^{[16]}$. Dissolution profile of EFA was improved by co-micronization with sodium lauryl sulphate and polyvinylpyrrolidone ${ }^{[17]}$. In the present study, EFA liposomes were prepared using thin film hydration technique by Box-Behnken design and assessed for vesicle size, entrapment efficiency (EE), drug release, in vitro drug release, bioavailability study, surface morphology. Solid liposomes were characterized by differential scanning calorimetry (DSC) and Fourier-transform infrared spectroscopy (FTIR).

\section{MATERIALS AND METHODS}

EFA was obtained as a gift from Mylan Laboratories, Nasik, India. Soya lecithin (SL), cholesterol, PEG
400 were purchased from S. D. Fine-Chem Limited, Mumbai, India. Chloroform, methanol and pepsin were purchased from Loba Chemicals. Dialysis bag was purchased from Analab Fine Chemicals. All the chemicals used were of analytical grade.

\section{Preliminary studies:}

Solubility studies were carried out using shake flask method $^{[18]}$. Different amounts of SL i.e. 500, 600, $700,800,900 \mathrm{mg}$ with excess amount of drug in 100 $\mathrm{ml}$ distilled water were placed in an orbital shaker (Make: REMI, Model: CRS-24BL) at $37^{\circ}$ for $24 \mathrm{~h}$. The suspensions were filtered through Whatman filter paper no 1 and filtrates were analysed by UV/Vis spectrophotometer at $\lambda \max 247 \mathrm{~nm}$.

\section{Preparation of blank liposomes:}

Preliminary studies involved preparation of liposomes using SL and cholesterol by thin film hydration method. Different ratios of SL:cholesterol was used i.e. 5:5, 6:4, 7:3, 8:2, 9:1. These five liposomes were evaluated for vesicle size and zeta potential. Besides this different batches of liposomes were prepared with different ratios of total lipid blend and drug (TLB:D), which included 4:1, 6:1 and 8:1. These liposomes were evaluated for vesicle size and zeta potential. Liposomes containing PEG 400 at concentrations of 5, 10 and $15 \% \mathrm{v} / \mathrm{v}$ were also prepared to investigate its effect on the vesicle size and entrapment efficiency ${ }^{[19]}$.

\section{Preparation of drug-loaded liposomes:}

Based on preliminary trials drug-loaded liposomes were prepared. Briefly, SL and cholesterol were dissolved in chloroform along $200 \mathrm{mg}$ of EFA and the chloroform was evaporated by hand-shaking the solution in a round bottom flask at room temperature to form a dry film. The film was hydrated with $10 \mathrm{ml}$ of phosphate buffer solution ( $\mathrm{pH}$ 7.4) with a predetermined quantity of PEG 400. A milky liposomal dispersion was formed and was subjected to size of vesicles and stored at $4^{\circ}$ until further evaluation ${ }^{[20]}$.

\section{Experimental design:}

From preliminary studies, effect of three independent variables at three different levels on various responses was evaluated using Box-Behnken design. These variables included TLB:D (A), SL:cholesterol (B) and PEG 400 (C) with vesicle size (Y1) and EE (Y2) as responses. Based on the 3 levels given in Table 1, 13 trial batches (R1-R13) were generated by Design Expert software (Design Expert 10, Stat-Ease, 
Minneapolis, MN; Table 2). The measured responses were used to construct $3 \mathrm{D}$ response surface plots to establish the relationship between variables and their interactions ${ }^{[21,22]}$. Analysis of variance (ANOVA) was used to validate the design. The optimized batch was selected on the basis of high EE and vesicle size. Drug release studies were also performed for these batches.

\section{Solidification of liposomes:}

Solidification of optimized liposomal dispersion was done by adsorption method ${ }^{[23]}$ wherein different adsorbents like aerosil, microcrystalline cellulose, calcium carbonate, magnesium carbonate and $\beta$-CD nanosponges were evaluated. $\beta$-CD-based nanosponges was prepared using diphenyl carbonate as crosslinker ${ }^{[16]}$. Liposomal dispersion and adsorbent material were mixed in the ratios of 1:1, 1:2, 1:3 and $1: 4$ till a free-flowing solid mass was obtained. Weighed amount of adsorbent was taken in porcelain mortar and $1 \mathrm{ml}$ of dispersion was added drop-wise and triturated to obtain a homogenous mass ${ }^{[24]}$.

\section{Characterization of liposomal dispersion:}

The mean vesicle size, vesicle size distribution and zeta potential of the Box-Behnken batches were analysed by Malvern Zeta Sizer (model: NanoZS90) based on the principle of dynamic light scattering. Liposomal dispersion $(1 \mathrm{ml})$ was diluted 10 times with doubledistilled water. An average electric field of about

\begin{tabular}{|c|c|c|c|}
\hline Coded value & TLB:D & SL:Ch & PEG-400 \\
\hline-1 & $4: 1$ & $5: 5$ & $5 \%$ \\
\hline 0 & $6: 1$ & $7: 3$ & $10 \%$ \\
\hline 1 & $8: 1$ & $9: 1$ & $15 \%$ \\
\hline
\end{tabular}

TLB:D is total lipid blend:drug, SL:Ch is soya lecithin:cholesterol, PEG 400 is polyethylene glycol 400

\section{TABLE 2: RESPONSES IN BOX-BEHNKEN DESIGN}

\begin{tabular}{lccccc}
\hline $\begin{array}{l}\text { Batch } \\
\text { codes }\end{array}$ & $\begin{array}{c}\text { TLB:D } \\
(\mathbf{m g})\end{array}$ & $\begin{array}{c}\text { SL:Ch } \\
(\mathbf{m g})\end{array}$ & $\begin{array}{c}\text { PEG } 400 \\
(\mathbf{m g})\end{array}$ & $\begin{array}{c}\text { Particle } \\
\text { size }(\mathrm{nm})\end{array}$ & $\begin{array}{c}\text { Entrapment } \\
\text { efficiency }(\%)\end{array}$ \\
\hline R1 & -1 & 0 & -1 & $1200 \pm 25$ & $90.87 \pm 2.5$ \\
R2 & 0 & 0 & 0 & $941 \pm 17$ & $83.57 \pm 2.8$ \\
R3 & 1 & 0 & 1 & $694.7 \pm 36$ & $96.08 \pm 3.7$ \\
R4 & -1 & 0 & 1 & $995.4 \pm 22$ & $95.53 \pm 1.9$ \\
R5 & 1 & -1 & 0 & $1088.0 \pm 38$ & $87.08 \pm 2.4$ \\
R6 & -1 & -1 & 0 & $857.8 \pm 25$ & $85.87 \pm 2.8$ \\
R7 & 1 & 0 & -1 & $1046.0 \pm 39$ & $85.82 \pm 2.8$ \\
R8 & -1 & 1 & 0 & $1178 \pm 28$ & $84.58 \pm 1.9$ \\
R9 & 0 & 1 & 1 & $886.8 \pm 24$ & $90.75 \pm 1.6$ \\
R10 & 1 & 1 & 0 & $1009.0 \pm 31$ & $97.54 \pm 2.1$ \\
R11 & 0 & 1 & -1 & $878.3 \pm 27$ & $87.29 \pm 2.6$ \\
R12 & 0 & -1 & -1 & $1074.0 \pm 26$ & $94.73 \pm 2.1$ \\
R13 & 0 & -1 & 1 & $706.6 \pm 25$ & $91.66 \pm 2.4$ \\
\hline
\end{tabular}

TLB:D is total lipid blend:drug, SL:Ch is soya lecithin:cholesterol, PEG 400 is polyethylene glycol $400, n=3$, mean $\pm S D$
16.25 V/cm and average current of about $0.05 \mathrm{~mA}$ were applied for measurement of zeta potentia ${ }^{[25]}$.

EFA entrapped within the liposomes was estimated after removing the unentrapped drug. The unentrapped drug was separated by subjecting the dispersions to centrifugation (Remi, RM-12CBh) at $7000 \mathrm{rpm}$ for $20 \mathrm{~min}$ whereupon liposomes settle at the bottom in the form of sediment and the supernatant containing free drug was collected, diluted with methanol and analysed for drug content by measuring absorbance at $247 \mathrm{~nm}$ using UV spectrophotometer ${ }^{[26]}$. EE for all the 13 batches was calculated using the formula, \% $\mathrm{EE}=$ (total drug-untrapped drug)/(total drug) $\times 100$.

The morphology of optimized EFA-loaded liposome was visualized under transmission electron microscopy (TEM, TECNAI G2F-20). The liposomal formulation (4 $\mathrm{mg} / \mathrm{ml}$ ) was diluted with double-distilled water before its placement on a copper grid. A $2 \%$ solution of phosphotungstic acid was added as a staining agent ${ }^{[27]}$. After drying at room temperature, the thin film was observed under the TEM.

\section{In vitro drug release:}

The in vitro release studies of drug from the liposomal formulations was carried out using USP type II dissolution apparatus by using dialysis membrane of molecular weight 12000 Da (HiMedia Laboratories, Mumbai) in simulated gastric fluid (SGF) for $2 \mathrm{~h}$ and in simulated intestinal fluid (SIF) for next $4 \mathrm{~h}$. Dialysis membrane was soaked overnight in $10 \mathrm{M}$ phosphate buffer prior to use ${ }^{[28]}$. A pouch was fashioned out of the dialysis membrane and $1 \mathrm{ml}$ of the liposomal dispersion containing approximately $20 \mathrm{mg}$ of EFA was placed in it. This was tied to the paddle of USP type 2 apparatus with $100 \mathrm{ml}$ of dissolution media at $100 \mathrm{rpm}$ at $37^{\circ}$. Aliquots $(3 \mathrm{ml})$ of dissolution media were withdrawn at different time intervals and the sample was replaced with fresh SGF and SIF to maintain constant volume. The samples were analysed by UV spectrophotometer at a $\lambda \max$ of $247 \mathrm{~nm}^{[29]}$.

\section{Evaluation of solid liposomes:}

Drug content was determined by dissolving $100 \mathrm{mg}$ of solid liposomes in ethanol with shaking. EFA content in the solution was assayed by measuring the absorbance at $247 \mathrm{~nm}$ using a UV spectrophotometer. Drug content $=$ (practical drug content $) /($ theoretical drug content $) \times 100$. The powder blends were evaluated for various micromeritic properties such as angle of repose, Hausner's ratio and Carr's index ${ }^{[30]}$. 
Thermograms were obtained for plain drug and optimized EFA-loaded liposomes using DSC (Mettler Toledo, DSC 823e). The heating rate of $10^{\circ} / \mathrm{min}$ was employed in the $30-300^{\circ}$ temperature range. Standard aluminium pans were used, an empty pan was used as reference standard. Analysis was performed on $5 \mathrm{mg}$ sample under nitrogen purge $(40 \mathrm{ml} / \mathrm{min})^{[31]}$.

The IR spectrum of optimized EFA-loaded liposomes was recorded using FTIR spectrophotometer (Jasco FTIR-460+spectrophotometer) with diffuse reflectance principle. Sample preparation involved mixing the sample with potassium bromide $(\mathrm{KBr})$, triturating in the glass mortar and finally placing in the sample holder. The spectrum was scanned over a frequency range $4000-400 \mathrm{~cm}^{-1[32]}$.

\section{Stability studies:}

The optimized liposomal dispersion and solid liposomes were placed in a light-shielded vials and stored in a stability chamber at $40^{\circ}$ and $75 \% \mathrm{RH}$ for 3 mo (as per ICH guidelines). At one-mo time intervals, samples were withdrawn, dissolved in methanol and checked for vesicle size, IR spectra and drug content ${ }^{[33]}$.

\section{Bioavailability studies:}

Approval for animal studies was granted by the Institutional Animal Ethics Committee, with the approval number, CPCSEA/IAEC/PT-02/02-2017. These studies were carried out as per the guidelines of Organization for Economic Co-operation and Development and Committee for the Purpose of Control and Supervision of Experiments on Animals. Animal studies were conducted using male Wistar rats to demonstrate the bioavailability of a drug. The animals were fasted overnight $(12 \mathrm{~h})$ prior to dosing. Plain vehicle, optimized liposomal dispersion, aqueous dispersion of solid liposomes and aqueous drug suspension (dose: $0.11 \mathrm{mg} / \mathrm{g}$ ) were administered to four groups of six rats in each with the aid of oral feeding needle. Blood samples $(2 \mathrm{ml})$ were withdrawn from the retro-orbital plexus at $0,2,4$ and $6 \mathrm{~h}$ intervals and collected in EDTA-coated tubes. The blood samples were centrifuged immediately at $1500 \mathrm{rpm}$ for $10 \mathrm{~min}$ and the separated plasma was stored at $-20^{\circ}$ in screwcapped polypropylene tubes till the time of analysis ${ }^{[34]}$. Analysis of the plasma samples to quantify the drug in the plasma were done using reversed-phase highperformance liquid chromatography method using methanol:0.1 $\mathrm{mM}$ dipotassium hydrogen phosphate buffer $(90: 20 \% \mathrm{v} / \mathrm{v})$ as mobile phase at flow rate of
$1 \mathrm{ml} / \mathrm{min}$. Injection volume was $20 \mu \mathrm{l}$ and UV/Vis SPD 20A detector was used at wavelength of $247 \mathrm{~nm}$. Column used was Agilent TC C18 $(250 \times 4.6 \mathrm{~mm}$ i.d., 5 $\mu \mathrm{m}$ vesicle size $)^{[35]}$.

\section{RESULTS AND DISCUSSION}

Solubility studies were carried out to find different concentration of SL for preparing liposomes. As EFA is hydrophobic (log P- 5.4) it was found to be soluble in SL. Since, SL and cholesterol were the key components of liposomes, it was necessary to determine their proportion. Cholesterol imparts rigidity to the liposomal membrane and SL forms the membrane and is responsible for improving solubility of drug. Cholesterol is not responsible for enhancement of drug solubility; hence no solubility studies were carried out with cholesterol. Various concentrations of SL were studied (Table 3). An increase in solubility of EFA was observed with increasing concentration of SL up to $700 \mathrm{mg}$. No significant increase in solubility of EFA was evident in 800 and $900 \mathrm{mg}$ of SL. Based on these results further studies were carried out to identify SL and cholesterol ratio to prepare blank liposomes.

Liposomes without drug were prepared using thin film hydration method with different ratios of SL:cholesterol (Table 4). The vesicle size of the liposomes ranged from 241.1 to $602.1 \mathrm{~nm}$. Zeta potential of the liposomes ranged from -1.33 to $-8.96 \mathrm{mV}$. SL:cholesterol ratio of 5:5 gave liposomes of least vesicle size. However equal concentrations of SL and cholesterol would cause extensive retardation of drug release as the bilayer becomes very inflexible ${ }^{[36]}$. Liposomes of different ratios of TLB:D were also prepared and evaluated for vesicle size (Table 4). The size of the drug-loaded liposomes ranged from 473.9 to $609.4 \mathrm{~nm}$ and zeta potential ranged from -9.86 to $17.6 \mathrm{mV}$. Least vesicle size was observed at TLB:D ratio of 6:1. It was however observed that loading of drug into the liposomes brought about a significant increase in size compared to the size of blank liposomes. It could be presumed that EFA being lipophilic in nature, got

TABLE 3: SOLUBILITY STUDIES

\begin{tabular}{lcc}
\hline Components & $\begin{array}{c}\text { Solubility } \\
(\mu \mathrm{g} / \mathrm{ml})\end{array}$ & $\begin{array}{c}\text { Solubility } \\
\text { enhancement } \\
\text { ratio }\end{array}$ \\
\hline Drug+water & $8.45 \pm 1.56$ & -- \\
Drug+SL $(500 \mathrm{mg})+$ water & $10.32 \pm 2.15$ & 1.22 \\
Drug+SL $(600 \mathrm{mg})+$ water & $11.11 \pm 1.98$ & 1.31 \\
Drug+SL $(700 \mathrm{mg})+$ water & $26.52 \pm 2.58$ & 3.13 \\
Drug+SL $(800 \mathrm{mg})+$ water & $26.67 \pm 2.66$ & 3.15 \\
Drug+SL $(900 \mathrm{mg})+$ water & $27.82 \pm 2.55$ & 3.29 \\
\hline
\end{tabular}

$S L$ is soya lecithin; $n=3$, mean $\pm S D$ 
TABLE 4: EVALUATION OF BLANK LIPOSOMES

\begin{tabular}{lcc}
\hline Parameters & \multicolumn{2}{c}{ Responses } \\
\hline SL:Ch & Particle size $(\mathrm{nm})$ & Zeta potential $(\mathrm{mV})$ \\
\hline $5: 5$ & $241.1 \pm 0.08$ & $-6.89 \pm 1.2$ \\
$6: 4$ & $416 \pm 0.50$ & $-1.75 \pm 0.5$ \\
$7: 3$ & $367 \pm 0.32$ & $-1.33 \pm 0.8$ \\
$8: 2$ & $602.1 \pm 0.42$ & $-8.13 \pm 1.58$ \\
$9: 1$ & $437 \pm 1.36$ & $-8.96 \pm 1.98$ \\
TLB:D & & \\
$4: 1$ & $583.7 \pm 1.65$ & $-9.16 \pm 1.3$ \\
$6: 1$ & $473.9 \pm 0.78$ & $-10.5 \pm 1.8$ \\
$8: 1$ & $609.4 \pm 0.76$ & $-17.6 \pm 2.5$ \\
PEG 400 & & \\
$5 \%$ & $425.8 \pm 0.65$ & $-12.1 \pm 1.6$ \\
$10 \%$ & $398.9 \pm 0.79$ & $-18.7 \pm 1.7$ \\
$15 \%$ & $497.0 \pm 0.32$ & $-13.5 \pm 2.1$ \\
\hline
\end{tabular}

TLB:D is total lipid blend:drug, SL:Ch is soya lecithin:cholesterol, PEG 400 is polyethylene glycol $400, n=3$, Mean \pm SD

interspersed in the lipid bilayer, which increased the vesicle diameter. Hence further studies were conducted by including PEG 400 in the liposomes with different TLB:D ratios. Based on the results of the preliminary studies, different ratios of SL:cholesterol, TLB:D and PEG 400 concentration were selected for further studies (Table 1).

Box-Behnken design with 3 factors at 3 different levels was used to investigate effect of independent variables on some key responses. Thin film hydration method was used to prepare 13 batches, which were evaluated for vesicle size and percent EE (Table 2). Analysis of experimental results was performed using the StatEase Design Expert version 10.0.

Regression analysis for response Y1 (vesicle size) is shown in Eqn. 1. The vesicle size for the formulations prepared as per Box-Behnken design varied from 694.7 to $1200 \mathrm{~nm}$ (Table 2). The polynomial Eqn. (Eqn. 1) generated by the software after analysis suggested that factor A (TLB:D), factor B (SL:cholesterol) and factor $\mathrm{C}$ (PEG 400) had positive effects on vesicle size. Hence, these results might show that vesicle size increased with increase in TLB:D ratio and concentration of PEG 400. The same is reflected in the response surface diagram shown in fig. 1A. As discussed earlier EFA, being lipophilic in nature, appeared to be located in the lipid bilayer, thus resulting in increased vesicle size. Ramana et al. reported an increase in liposome size with increase in drug to lipid ratio for nevirapine ${ }^{[37]}$. Liposome surface being hydrophilic in nature has a hydration layer surrounding each vesicle. The PEG 400 molecules get interspersed in the hydration layer, which results in additional surface hydration thickness and thereby increase in size of vesicle. However, the PEG molecules stabilize the vesicle size by preventing aggregation of vesicles ${ }^{[38]}$. Eqn. $1, \quad \mathrm{PS}=941.50+79.66 \mathrm{~A}+55.81 \mathrm{~B}+113.28 \mathrm{C}-$ $20.00 \mathrm{AB}+140.82 \mathrm{AC}+118.97 \mathrm{BC}+135.62 \mathrm{~A}^{2}+35.87 \mathrm{~B}^{2}-$ $65.95 \mathrm{C}^{2}$. The model $\mathrm{F}$ value of 5.43 implied that the quadratic model was significant. Values of 'prob $>F$ ' less than $0.0500(0.0182)$ indicated that model terms were significant. In this case factor $\mathrm{A}$ and $\mathrm{C}$ were significant model terms.

Regression analysis for response Y2 (\% EE) is shown in Eqn. 2. The \% EE varied from 99.87-94.73\% (Table 2). It is clear from the Eqn. that the factor A (TLB:D) had a positive effect on \% EE. Therefore, increase in TLB:D ratio increased the drug EE. This was confirmed by the probability values (p-0.0127) generated by ANOVA studies. The $p$ value $(0.0150)$ for the term $\mathrm{C}^{2}$ is indicative of an exponential relationship between EE and concentration of PEG 400. Besides stabilizing the vesicle size, PEG 400 also acted as a solubilizer for EFA. Chika et al. reported a 16-fold increase in aqueous solubility of EFA in the presence of PEG $400^{[39]}$. PEG 400 might have increased the EE by enhancing the solubility of EFA in the aqueous inner phase of the liposomes. Similar findings were reported for paclitaxel due to inclusion of PEG 400 in the liposomal formulation ${ }^{[40]}$. Thus, EFA could be partitioned in the lipid bilayer as well as the aqueous inner core leading to increased $\mathrm{EE}$. The response surface diagram for $\mathrm{EE}$ is presented in fig. 1B. Eqn. 2, $\mathrm{EE}=$ $97.58+1.16 \mathrm{~A}-0.63 \mathrm{~B}-0.084 \mathrm{C}-0.47 \mathrm{AB}-0.32 \mathrm{AC}+0.22$ $\mathrm{BC}+0.036 \mathrm{~A}^{2}-0.38 \mathrm{~B}^{2}+0.93 \mathrm{C}^{2}$. The Model F-value of 6.17 implied that the model was significant. In this case $\mathrm{A}, \mathrm{B}, \mathrm{C}^{2}$ were significant model terms. Importantly, increase in the cholesterol content did not enhance drug loading capacity of the liposomes. Instead it reduced the encapsulation efficiency. High levels of cholesterol have been reported to interfere with the close packing of lipids in the vesicles ${ }^{[36]}$. The encapsulation of EFA in the liposomes was confirmed by DSC measurements. High EE was one of the critical factors used to express the quality of liposomal formulation in the therapeutic effect of drugs. The drug lipid ratio is a critical formulation parameter, which affects number of vesicular properties including release profile, EE and vesicle size. Different drug-lipid ratios were studied to regulate the release of doxorubicin form the vesicles ${ }^{[41]}$. This ratio can be used as an index of the effectiveness of the preparation method too. Furthermore, druglipid ratio influenced the therapeutic efficacy of the liposomal product, expressing the actual dose of the drug being administered ${ }^{[42]}$. 


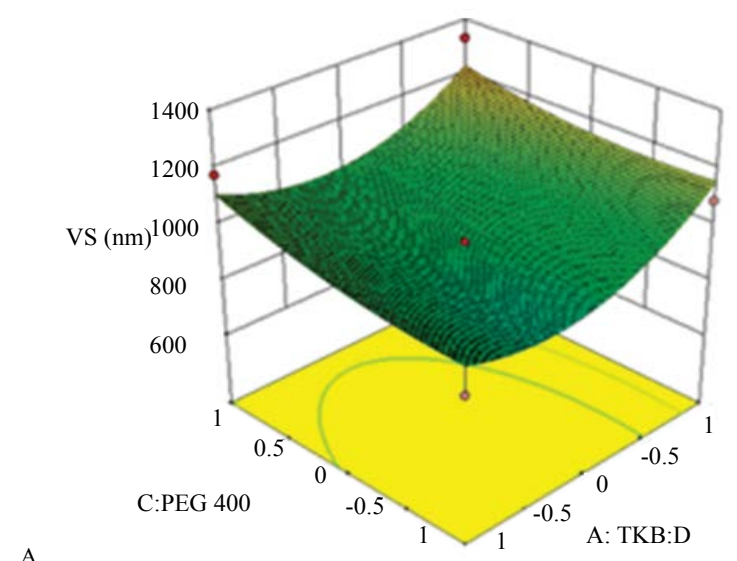

A.

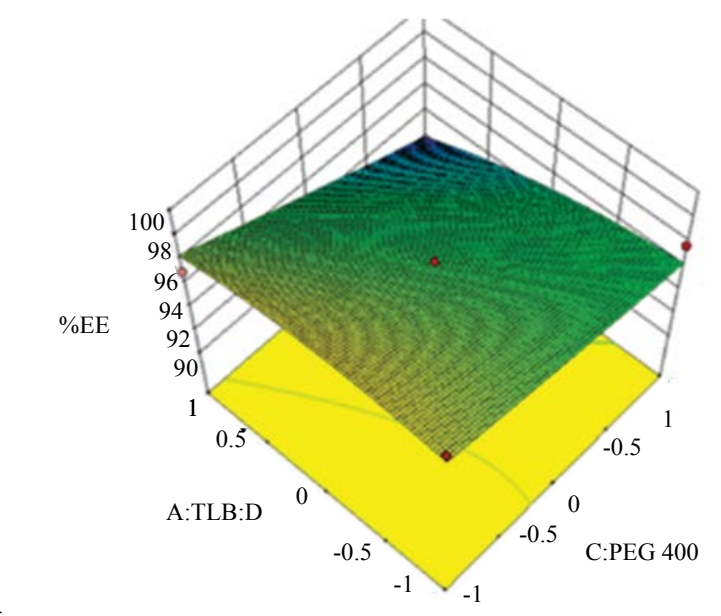

Fig. 1: 3D response surface of A. vesicle size and B. \% EE

Among the various formulations, optimal formulations were selected on the basis of responses of maximum $\mathrm{EE}$ and in range vesicle size. The software generated 50 solutions, among which one was chosen on the basis of highest desirability factor. The optimized formulation was prepared with TLB:D ratio of 4:1, SL:cholesterol ratio of $7: 3$ and PEG 400 at $10 \% \mathrm{v} / \mathrm{v}$ concentration. As TLB consisted of SL and cholesterol, the TLB amount is distributed in 7:3 proportion with constant amount of drug. The optimized formulation was evaluated for vesicle size, EE, in vitro drug release, TEM studies and pharmacokinetic studies.

The vesicle size of optimized formulations was found to be $945.6 \mathrm{~nm}$. Liposomes were prepared using thin film hydration method, which resulted in the formation of large multilamellar and unilamellar vesicles as explained by Yadav et al. ${ }^{[33]}$. Polydispersity is the ratio of standard deviation to mean droplet size; hence, it indicates the uniformity of droplet size within the formulation. The higher the polydispersity, the lower the uniformity of the droplet size in the formulation. The closer to zero the polydispersity value reached, the more homogenous were the droplets. The polydispersity index was found to be 0.487 , which indicated homogenous nature of the particles.

The zeta potential value of optimized formulation was $-10.6 \mathrm{mV}$. The results of the vesicle size and zeta potential supported the acceptable physical stability as high absolute zeta potential values tend to hinder the probability of coalescence and thereby maintain the homogeneity of vesicle sizes. The zeta potential value between +30 to $-30 \mathrm{mV}$ imparts good stability to the liposomal encapsulation. EE of optimized formulation was found to be $98.92 \%$.

The optimized liposomal dispersion was subjected to TEM studies to determine the inner structure of the liposomes. Fig. 2 shows the lipid bilayer and the aqueous compartment. TEM image showed that multi-vesicular and multi-lamellar liposomes were formed after hydration ${ }^{[43]}$. In the TEM image for the EFA-loaded liposome, the droplets were observed as spherical in shape and liposomal formulation was a monodispersed stable system and could deliver the drug effectively.

The findings of the in vitro dissolution profile in SGF and SIF clearly showed that the dissolution rate of EFA was significantly improved after the encapsulation. Percent release values of the drug over $6 \mathrm{~h}$, in SGF for $2 \mathrm{~h}$ and in SIF for $4 \mathrm{~h}$ determined for the Box-Behnken design batches of the liposomal dispersions are shown in fig. 3. In vitro release ranged from a lowest of $20.75 \%$ for batch R5 to a maximum cumulative release of $93.03 \%$ for batch R1 in a span of $6 \mathrm{~h}$ (Table 5). Plain EFA showed less than $10 \%$ release in $6 \mathrm{~h}$. The drug is present in solubilized form in the lipid bilayer as well as the aqueous inner liposomal core. Its diffusion through the liposome seems to be facilitated by the formation

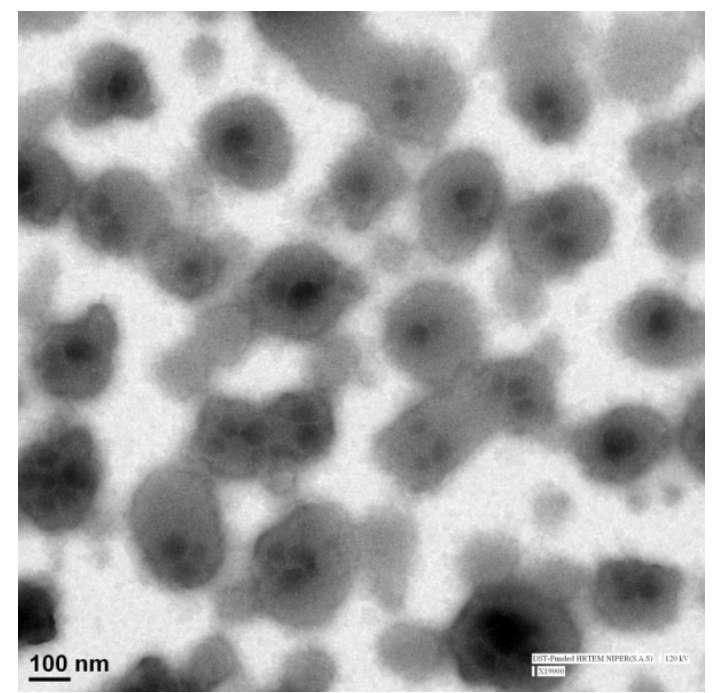

Fig. 2: TEM image of liquid liposomes 


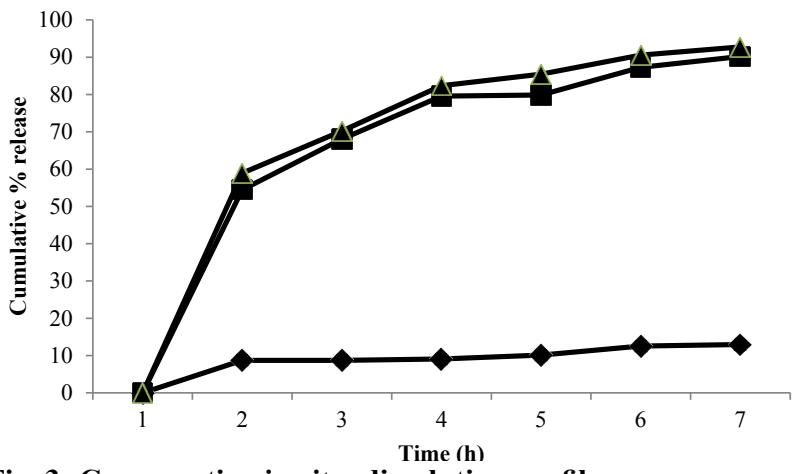

Fig. 3: Comparative in vitro dissolution profile

(一४) Plain drug, (一匹-) optimized solid liposomes and (一 - ) optimized liposomal dispersion

TABLE 5: IN VITRO DRUG RELEASE STUDIES

\begin{tabular}{lcc}
\hline Batches & $\begin{array}{c}\text { Cumulative } \\
\text { percent drug } \\
\text { release in SGF in } \\
2 \mathrm{~h}\end{array}$ & $\begin{array}{c}\text { Cumulative } \\
\text { percent drug } \\
\text { release in SIF in } \\
6 \mathrm{~h}\end{array}$ \\
\hline R1 & $31.07 \pm 0.68$ & $93.03 \pm 0.16$ \\
R2 & $20.84 \pm 0.23$ & $37.89 \pm 0.53$ \\
R3 & $18.36 \pm 0.31$ & $28.86 \pm 0.28$ \\
R4 & $16.65 \pm 0.63$ & $21.65 \pm 0.26$ \\
R5 & $15.21 \pm 0.54$ & $20.75 \pm 0.43$ \\
R6 & $16.65 \pm 0.32$ & $21.32 \pm 0.91$ \\
R7 & $16.07 \pm 0.54$ & $27.03 \pm 0.31$ \\
R8 & $42.24 \pm 0.21$ & $76.60 \pm 0.56$ \\
R9 & $20.25 \pm 0.82$ & $80.97 \pm 0.79$ \\
R10 & $31.43 \pm 0.72$ & $58.32 \pm 0.81$ \\
R11 & $37.46 \pm 0.56$ & $91.42 \pm 0.56$ \\
R12 & $47.25 \pm 0.89$ & $88.63 \pm 0.15$ \\
R13 & $18.81 \pm 0.91$ & $61.83 \pm 0.32$ \\
Plain EFA & $5.72 \pm 0.72$ & $9.5 \pm 0.62$ \\
Optimized & & \\
liposomal & $25.65 \pm 0.76$ & $94.28 \pm 0.75$ \\
dispersion & & \\
Optimized solid & $28.07 \pm 0.32$ & $93.12 \pm 0.12$ \\
liposomes &
\end{tabular}

$\mathrm{n}=3$, mean $\pm \mathrm{SD}$

of transient pores in the lipid bilayer, through which drug was released to the extra-liposomal medium ${ }^{[44]}$. Higher concentration of cholesterol appeared to cause rigidity of the lipid bilayer resulting in smaller and lesser number of pores. The hydroxyl group of cholesterol formed hydrogen bonds with the carbonyl groups of soya lecithin causing rigidity of the liposomal bilayer and effectively reducing the pore size ${ }^{[45]}$. Thus, formulations containing higher amount of cholesterol showed significantly slower release. The optimized liposomal dispersion displayed $94.28 \%$ cumulative drug release in $6 \mathrm{~h}^{[46]}$.

The optimized liposomal formulation was converted into solid form by adsorption onto different adsorbents like aerosil, MCC and $\beta$-CD NS at different ratios of $1: 1,1: 2$ and 1:3. The blend, which produced a freeflowing powder was selected for further studies. The angle of repose for different ratios of aerosil ranged from $30-32^{\circ}$ while for MCC the values ranged from $34-37^{\circ}$. The blend with $\beta-C D$ nanosponges displayed excellent flow properties with angle of repose in the range of $27-30^{\circ}$. The $1: 2$ ratio of optimized liposome with $\beta$-CD nanosponge was selected for further investigations as it showed least angle of repose. The nanosponges have several nanocavities created due to cross linking of $\beta-C D$ with diphenyl carbonate, which act as potential sites for entrapment of hydrophilic and hydrophobic moieties along with the $\beta$-CD cavities themselves ${ }^{[47]}$. Thus, the liposomes are presumable getting encapsulated within these cavities and nanochannels. The drug content for solid optimized formulation was $98.02 \%$, which was nearly equal to EE.

DSC thermograms of EFA exhibited a sharp melting endothermic peak at $140.16^{\circ}$ (fig. 4) showing polymorphic form 1. Radesca et al. claimed that EFA exists in polymorphic form 1 (crystalline), polymorphic forms 2 and 3 show peak at $140^{\circ}, 116^{\circ}$ and $108^{\circ}$ respectively where form 1 shows therapeutic efficacy in strengths of 50, 100 and $200 \mathrm{mg}^{[48]}$. Liposomeloaded nanosponges did not show any endothermic peak of EFA. Hence, it could be concluded that EFA was encapsulated into the nanosponges ${ }^{[49]}$. This has also indicated a change in the physical nature of EFA from the crystalline state to the amorphous one.

The IR spectra of EFA and solid liposomes were recorded. The characteristic peaks observed for EFA include - $\mathrm{NH}$ stretch, $\mathrm{C}=\mathrm{O}$ stretch, $\mathrm{C}-\mathrm{F}$ stretch and $\mathrm{C}-\mathrm{Cl}$ stretch at 3317.3, 1749.7, 1496.9 and $1038.3 \mathrm{~cm}^{-1}$, respectively ${ }^{[50]}$. The FTIR spectra of solid liposomes showed a broad peak at $3400 \mathrm{~cm}^{-1}$ due to $\mathrm{OH}$ groups present in cholesterol, which is part of the bilayer as well

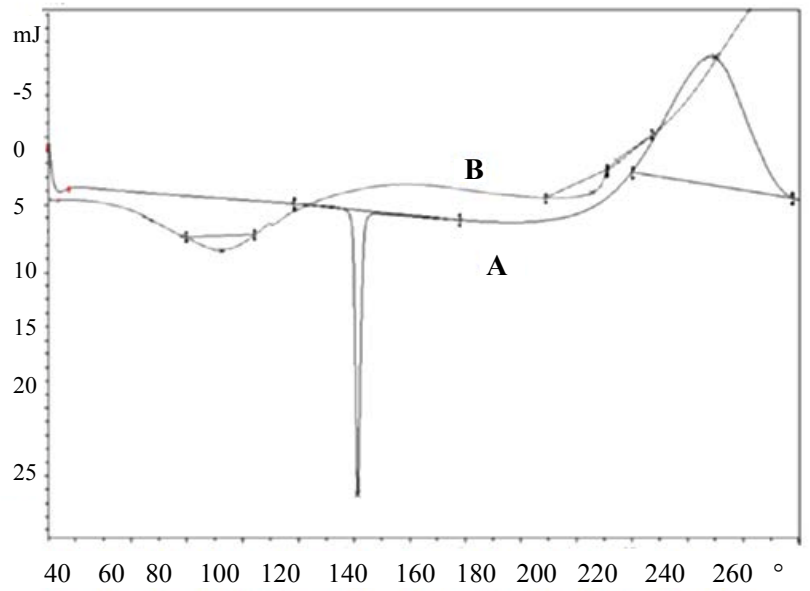

Fig. 4: DSC graph of A. plain EFA and B. solid liposomes Integral: $299.86 \mathrm{~mJ}$; peak: $140.16^{\circ}$ 
as the nanosponges. A peak at $1741 \mathrm{~cm}^{-1}$ is characteristic of the carbonate ester group both in $\beta-C D$ and EFA. The incorporation of drug in NS were further checked by drug content by UV spectrophotometer showing $\lambda_{\max }$ at $247 \mathrm{~nm}$ indicated that liquid formulation gets adsorbed on nanosponges (fig. 5).

Drug release studies were carried out for optimized solid liposomes and plain drug (fig. 3). After $6 \mathrm{~h}$, optimized solid liposomes showed $93.12 \%$ cumulative release while from unformulated drug the release was a meagre $9.5 \%$ in $6 \mathrm{~h}$. An insignificant decrease in release was observed with solid liposomes as compared to liquid liposomes as the liposomes were entrapped within NS cavities. Hence a delay in release from NS is expected.

Stability evaluation was carried out for 3 mo as per ICH guidelines and the vesicle size and EE was checked.

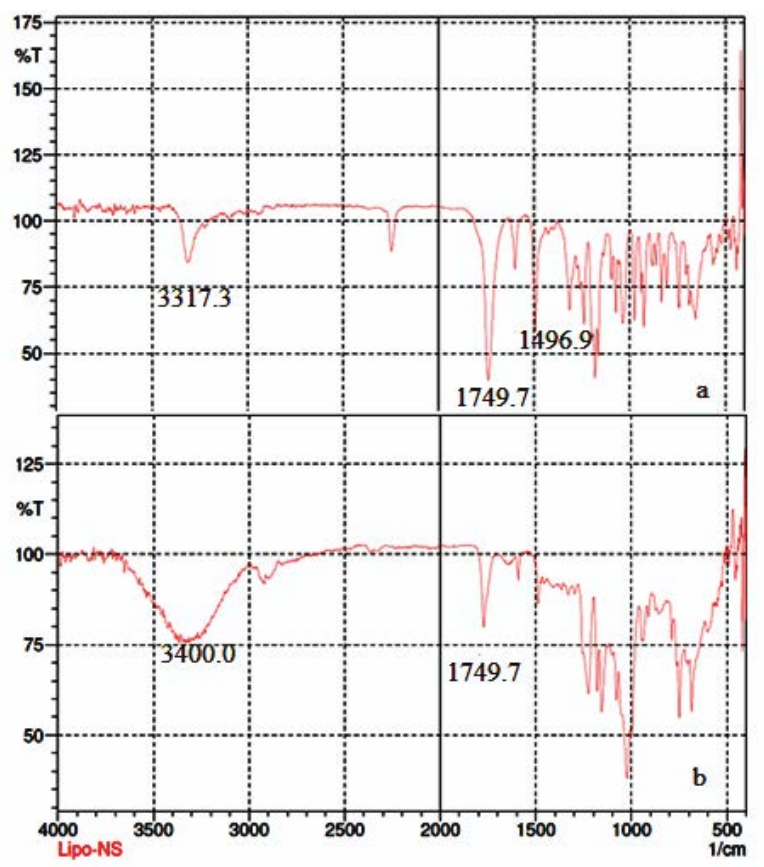

Fig. 5: FTIR-Spectra of a. EFA and b. solid liposomes

TABLE 6: MICROMERITICS PROPERTIES OF OPTIMIZED SOLID LIPOSOMES

\begin{tabular}{lcc}
\hline Properties & Values $^{*}$ & Significance \\
\hline Angle of repose & $27.85 \pm 1.26$ & Good \\
Hausner's ratio & $1.11 \pm 0.21$ & Excellent \\
Carr's index & $12.7 \pm 0.75$ & Good \\
\hline
\end{tabular}

$\mathrm{n}=3$, mean $\pm \mathrm{SD}$

TABLE 7: PHARMACOKINETIC PARAMETERS
An insignificant change in vesicle size and EE was observed for the optimized liposomal dispersion. Vesicle size after $3^{\text {rd }}$ mo was found to be $976.5 \mathrm{~nm}$ with EE of $98.32 \%$. Micromeritic properties of optimized solid liposomes are shown in Table 6. No change was observed in the IR spectra and drug content ${ }^{[51]}$.

The in vivo pharmacokinetic parameters for bioavailability enhancement of optimized formulation were studied using bioanalytical HPLC method. The Cmax for plain drug, optimized liquid formulation and aqueous dispersion of solid liposomes were found to be $2.6,4.9$ and $5.2 \mu \mathrm{g} / \mathrm{ml}$, respectively and Tmax was found to be $2 \mathrm{~h}$. About 2-fold increase in Cmax was found in EFA liposomal dispersion as compared to plain EFA (Table 7). The $\mathrm{AUC}_{0-6 \mathrm{~h}}$ for the optimized liposomal dispersion and solid liposomes was found to display 2.5 and 2.6-fold increase in bioavailability, which showed significant increase as compared to plain drug. GraphPad Prism 7 software was used to compute statistical measures. ANOVA test was used to find out significant differences in plain drug and formulation, the $\mathrm{P}$ value (0.0049) was below 0.01 indicating a significant difference in pharmacokinetics of test formulations.

The larger amount of dissolved drug encapsulated in the liposomes translated into higher oral bioavailability. As seen in the in vitro dissolution studies most of the drug is released in small intestine. The intestinal lymphatic system is responsible for the absorption of lipid-based formulations. In case of lipophilic drug, the degree of lymphatic transport was strongly correlated with the triglyceride content of the lymph. The pancreatic lipases cause structural breakdown of the liposomes leading to the formation of mixed micelles and vesicular carriers in presence of bile salts, which transport the drug and free phospholipids through the intestinal mucosa ${ }^{[52]}$. The free phospholipids stimulate production of chylomicrons by the glycerol-3-phosphate pathway on rough endoplasmic reticulum, and the 2-monoglycerol pathway on smooth endoplasmic reticulum. Thus, the lipophilic drug associated with chylomicrons is expected to enter intestinal lymphatics ${ }^{[53]}$. As EFA is highly lipophilic in nature $(\log \mathrm{P}=5.4)$ we may presume that the drug is absorbed via the lymphatic route.

\begin{tabular}{lccc}
\hline Pharmacokinetic Tparameter & Optimized liposomal dispersion Aqueous dispersion of solidified liposomes & Plain EFA \\
\hline Cmax $(\mu \mathrm{g} / \mathrm{ml})$ & $4.9 \pm 0.72$ & $5.2 \pm 0.56$ & $2.6 \pm 0.19$ \\
Tmax & $2 \mathrm{~h}$ & $2 \mathrm{~h}$ & $2 \mathrm{~h}$ \\
$\mathrm{AUC}_{0.6 \mathrm{~h}}$ & $76.41 \pm 3.58$ & $80.20 \pm 5.65$ & $30.41 \pm 7.38$ \\
$\mathrm{~K}_{\mathrm{el}}\left(\mathrm{h}^{-1}\right)$ & $0.340 \pm 0.08$ & $0.250 \pm 0.07$ & $0.199 \pm 0.08$ \\
\hline
\end{tabular}

$\mathrm{n}=3$, mean $\pm \mathrm{SD}$ 
Besides this the HIV, which causes AIDS, settles in lymphoid organs such as the spleen, thymus and lymph nodes. In the early stage of infection and throughout the latent stage, the virus replicates vigorously in lymphoid organs, meaning that lymphatic drug delivery can be advantageous in the treatment of AIDS ${ }^{[54,55]}$. Thus, the pharmacokinetic studies demonstrated that EFA-loaded liposomes could significantly upgrade the solubility and oral bioavailability of EFA as well as improve the therapeutic efficacy.

The present study demonstrated that liposomes enhanced oral bioavailability of a poorly soluble nonnucleoside reverse transcriptase inhibitor, EFA. The liposomes loaded with EFA were prepared using thinfilm hydration method. These liposomes were evaluated for vesicle size, EE, in vitro drug release and in vivo pharmacokinetics. Improvement in dissolution profile signified enhanced solubility. The oral bioavailability of the formulation in rats was also remarkably enhanced by almost 2-fold. It could be concluded that the solubility and bioavailability of EFA was significantly improved after liposomal encapsulation.

\section{Acknowledgments:}

The authors would like to thank Dr Ashwini Madgulkar, Principal, AISSMS College of Pharmacy, for providing necessary help and facilities to carry out the research work. The authors acknowledge Mylan Laboratories, Nashik (India) for providing EFA as a gift sample.

\section{Conflict of interest:}

There is no conflict of interest, financial or otherwise associated with this project.

\section{Financial support and sponsorship:}

Nil.

\section{REFERENCES}

1. Khadka P, Ro J, Kim H. Pharmaceutical particle technologies: An approach to improve drug solubility, dissolution and bioavailability. Asian J Pharm Sci 2014;9:304-16.

2. Iannazzo D, Pistone A, Romeo R, Giofrè SV. Nanotechnology Approaches for Antiretroviral Drugs Delivery. J AIDS HIV Infect 2001;1(2):1-13.

3. Mamo T, Moseman EA, Kolishetti N, Salvador-Morales C, Shi J, Kuritzkes DR, et al. Emerging nanotechnology approaches for HIV/AIDS treatment and prevention. Nanomedicine 2010;5(2):269-85.

4. Parboosing R, Maguire GEM, Govender P, Kruger HG. Nanotechnology and the Treatment of HIV Infection. Viruses 2012;4:488-520.
5. Swami H, Kataria MK, Bilandi A, Kour P, Bala S. Liposome: An art for drug delivery. Int J Pharma Sci 2015;5(2):523-30.

6. Arvind G, Shah S, Mule S, Prashanth P, Konda N. Formulation and Characterization of Amphotericin B Liposomes Prepared by Thin Film Hydration Method. Int J Pharm 2013;3(3):540-47.

7. Yang G, Zhao Y, Zhang Y, Dang B, Liu Y, Feng N. Enhanced Oral Bioavailability of Silymarin Using Liposomes Containing a Bile Salt: Preparation by Supercritical Fluid Technology and Evaluation in vitro and in vivo. Int $\mathrm{J}$ Nanomedicine 2015;10:6633-44.

8. Chen Y, Lu Y, Chen J, Lai J, Sun J, Hu F, et al. Enhanced bioavailability of the poorly water-soluble drug fenofibrate by using liposomes containing a bile salt. Int $\mathrm{J}$ Pharm 2009;376:153-60.

9. Chougule M, Padhi B, Misra A. Development of Spray Dried Liposomal Dry Powder Inhaler of Dapsone. AAPS PharmSciTech 2008;9(1):1-12.

10. Aditya NP, Chimote G, Gunalan K, Banerjee R, Patankar S, Madhusudhan B. Curcuminoids-loaded liposomes in combination with arteether protects against Plasmodium berghei infection in mice. Exp Parasitol 2012;131:292-9.

11. Marripati S, Umasankar K, Reddy PJ.A Review on Liposomes. Int J Res Pharma NanoSci 2014;3(3):159-69.

12. Tanaka R, Hanabusa H, Kinai E, Hasegawa N, Negishi M, Kato S. Intracellular Efavirenz Levels in Peripheral Blood Mononuclear Cells from Human Immunodeficiency Virus-Infected Individuals. Antimicrob Agents Chemother 2008;52(2):782-85.

13. Prathap B, Dey A, Rao GS, Vennela S, Hussain S. Method Development and Validation of Efavirenz in Bulk and Pharmaceutical Dosage Form by UV Spectrophotometer. IJIPSR 2013;4(1):269-73.

14. Reddy MS, Reddy NS, Reddy SM. Solubility Enhancement of Poorly Water-Soluble Drug Efavirenz by Solid Self Emulsifying Drug Delivery Systems. IJPRR 2014;3(4):20-8.

15. Gaur PK, Mishra S, Bajpai M, Mishra A. Enhanced Oral Bioavailability of Efavirenz by Solid Lipid Nanoparticles: In vitro Drug Release and Pharmacokinetics Studies. Biomed Res Int 2014;2014:363404.

16. Rao MRP, Shirsath C. Enhancement of Bioavailability of Non-Nucleoside Reverse Transcriptase Inhibitor Using Nanosponges. AAPS PharmSciTech 2017;18(5):1728-38.

17. da Costa MA, Seiceira RC, Rodrigues CR, Hoffmeister CRD, Cabral LM, Rocha HVA. Efavirenz Dissolution Enhancement I: Co-Micronization. Pharmaceutics 2013;5(1):1-22.

18. Lankalapalli S, Tenneti V, Adama R. Preparation and Evaluation of Liposome Formulations for Poorly Soluble Drug Itraconazole by Complexation. Pharm Lett 2015;7(8):1-17.

19. Schwendener RA, Schott H. Liposomes formulations of hydrophobic drugs. In: Weissig V, editor. Liposomes: Method and protocols, Volume 1: Pharmaceutical Nanocarriers. New York City, New York, United States: Humana Press; 2010. p. 129-38.

20. Shavi GV, Sreenivasa Reddy M, Raghavendra R, Nayak UY, Kumar AR, Deshpande PB, et al. PEGylated Liposomes of Anastrozole for Long-Term Treatment of Breast Cancer: in vitro and in vivo evaluation. J Liposome Res 2016;26(1):28-46. 
21. Narayan R, Singh M, Ranjan O, Nayak Y, Garg S, Shavi GV. Development of risperidone liposomes for brain targeting through intranasal route. Life Sci 2016;163:38-45.

22. Rane S, Prabhakar B. Optimization of Paclitaxel Containing pH Sensitive Liposomes By 3 Factor, 3 Level Box-Behnken Design. Indian J Pharm Sci 2013;75(4):420-6.

23. Joshi M, Pathak S, Sharma S, Patravale V. Solid microemulsion preconcentrate (NanOsorb) of artemether for effective treatment of malaria. Int J Pharm 2008;362:172-8.

24. Trotta F,Zanetti M, Cavalli R. Cyclodextrin-based nanosponges as drug carriers. Beilstein J Org Chem 2012;8:2091-99.

25. Curić A, Reul R, Möschwitzer J, Fricker G. Formulation optimization of itraconazole loaded PEGylated liposomes for parenteral administration by using design of experiments. Int J Pharm 2013;448(1):189-97.

26. Cavalcanti IM, Mendonça EA, Lira MC, Honrato SB, Camara $\mathrm{CA}$, Amorim RV, et al. The encapsulation of b-lapachone in 2-hydroxypropyl-b-cyclodextrin inclusion complex into liposomes: A physicochemical evaluation and molecular modeling approach. Eur J Pharm Sci 2011;44(3):332-40.

27. Almgren M, Edwards K, Karlsson G. Cryo transmission electron microscopy of liposomes and related structures. Colloids Surf A 2000;174:3-21.

28. Sambrook JF, Russell DW. Molecular cloning: a laboratory manual. 3rd ed. Cold Spring Harbor, New York, United States: Cold Spring Harbor Laboratory Press; 2001. p. 56-7.

29. Gupta AS, Kshirsagar SJ, Bhalekar MR, Saldanha T. Design and development of liposomes for colon targeted drug delivery. J Drug Target 2013;21(2):146-60.

30. Sinko PJ. Martin's Physical Pharmacy and Pharmaceutical sciences. 6th ed. Baltimore, MD, USA: Lippincott Williams and Wilkins; 2010. p. 465-7.

31. Panwar P, Pandey B, Lakhera PC, Singh KP. Preparation, characterization, and in vitro release study of albendazoleencapsulated Nano size liposomes. Int J Nanomedicine 2010;5:101-9.

32. Cabral ECM, Zollner RL, Santana MHA. Preparation and Characterization of Liposomes Entrapping Allergenic Proteins. Braz J Chem Engg 2004;21(2):137-46.

33. Yadav AV, Murthy MS, Shete AS, Sfurti S. Stability Aspects of Liposomes. Indian J Pharm Edu Res 2011;45(4):402-13.

34. Zhu Y, Wang M, Zhang J, Peng W, Firempong CK, Deng W, et al. Improved oral bioavailability of capsaicin via liposomal nanoformulation: preparation, in vitro drug release and pharmacokinetics in rats. Arch Pharm Res 2015;38(4):512-21.

35. de Sá Viana O, Medeiros FPM, Jr Grangeiro S, Albuquerque MM, La Roca Soares MF, Soares-Sobrinho JL, et al. Development and validation of a HPLC analytical assay method for efavirenz tablets: a medicine for HIV infections. Braz J Pharm Sci 2011;47(1):1-6.

36. Gao W, Hu CM J, Fang RH, Zhang L. Liposome-like Nanostructures for Drug Delivery. J Mater Chem B Mater Biol Med 2013;1(48):1-35.

37. Ramana LN, Sethuraman S, Ranga U, Krishnan UM. Development of a liposomal nano delivery system for nevirapine. J Biomed Sci 2010;17:57-70.

38. Mukherjee B, Patra B, Layek B, Mukherjee A. Sustained release of acyclovir from nano-liposomes and nano-niosomes: An in vitro study. Int J Nanomedicine 2007:2(2):213-25.

39. Chika M, Edwin O, Gerald U. Study on the influence of cosolvent and surfactant on solubilizationof efavirenz. Euro J Biomed Pharm Sci 2016;3(7):522-6.

40. Yang T, Cui FD, Choi MK, Lin H, Chung SJ, Shim CK, et al. Liposome Formulation of Paclitaxel with Enhanced Solubility and Stability. Drug Deliv 2007;14:301-8.

41. Johnston MJ, Edwards K, Karlsson G, Cullis PR. Influence of drug-to-lipid ratio on drug release properties and liposome integrity in liposomal doxorubicin formulations. J Liposome Res 2008;18(2):145-57.

42. Chountoulesi M, Naziris N, Pippa N, Demetzos C. The significance of drug-to-lipid ratio to the development of optimized liposomal formulation. $\mathrm{J}$ Liposome Res 2018;28(3):249-58.

43. Sabeti B, Noordine MIB, Javar HA, Davoudi ET, Kadivar A. Characterization of Diclofenac Liposomes Formulated with Palm Oil Fractions. Trop J Pharm Res 2014;13(2):185-90.

44. Wang Y, Wang S, Firempong CK, Zhang H, Wang M, Zhang Y, et al. Enhanced Solubility and Bioavailability of Naringenin via Liposomal Nanoformulation: Preparation and In vitro and In vivo Evaluations. AAPS PharmSciTech 2016;18(3):586-94.

45. DenizA, Sade A, Severcan F, Keskin D, Tezcaner A, Banerjee S. Celecoxib-loaded liposomes: effect of cholesterol on encapsulation and in vitro release characteristics. Biosci Rep 2010;30:365-73.

46. Chandrashekharn $\mathrm{AR}$, Jia CY, Dhanaraj SA. In vitro studies and evaluation of Metformin Marketed Tablet-Malyasia. J Appl Pharm Sci 2011;1(5):214-7.

47. Rao M, Bajaj A. Investigation of nanoporous colloidal carrier for solubility enhancement of Cefpodoxime proxetil. J Pharm Res 2012;5(5):2496-9.

48. Radesca LA, Maurin MB, Rabel SR, Moore JR, inventors; Bristol-Myers Squibb Pharma Co., assignee. Crystalline Efavirenz. United States patent US6673372B1. 1998 Jun 11.

49. Maheshkumar SS, Reddy KN, Goud PP, Kiranmayi N, Arvind G. Formulation and Characterization of Doxorubicin Hydrochloride Liposomes by Double Emulsion Method. Int Res J Pharm 2013;4(4):197-201.

50. Reddy NP, Yenumula P, Perika M, Akari A. FTIR Spectroscopy for Estimation of Efavirenz In Raw Material and Tablet Dosage Form. Int Curr Pharm J 2015;4(6):390-5.

51. Sebaaly C, Jraij A, Fessi H, Charcosset C, Greige-Gerges H. Preparation and Characterization of Clove Essential OilLoaded Liposomes. Food Chem 2015;178:52-62.

52. Bozzuto G, Molinari A. Liposomes as Nanomedical Devices. Int J Nanomedicine 2015; 10:975-99.

53. Kim H, Kim Y, Lee J. Liposomal Formulations for Enhanced Lymphatic Drug Delivery. Asian J Pharm Sci 2013;8:96-103.

54. Cai S, Yang Q, Bagby TR, Forrest ML. Lymphatic Drug Delivery Using Engineered Liposomes and Solid Lipid Nanoparticles. Adv Drug Deliv Rev 2011;63(10):901-8.

55. Yáñez JA, Wang SW, Knemeyer IW, Wirth MA, Alton KB. Intestinal lymphatic transport for drug delivery. Adv Drug Delivery Rev 2011;63:923-42. 